\title{
Priorización del mantenimiento mediante la determinación del número prioritario de riesgo, y el análisis de modos y efectos de fallos de una máquina de inyección de poliuretano de alta presión
}

Prioritization of maintenance by determining the priority number of risk, and analysis of modes and effects of failures of a high pressure polyurethane injection machine

Angel Daniel Larrea Moreano. ${ }^{1}$, Cristian David Redrobán Dilon. ${ }^{2}$ \& Antonio Gabriel Castillo Medina. ${ }^{3}$

Recibido: 24-04-2020 / Revisado: 13-05-2020 / Aceptado: 22-06-2020 / Publicado: 03-07-2020

\begin{abstract}
.
DOI: https://doi.org/10.33262/cienciadigital.v4i3.1353

Prioritization of activities is very important, to know the importance of performing a maintenance activity. The Modes Analysis Methodology and Failure Effects (AMEF), is a tool that allows us to prioritize the maintenance care for equipment; currently this tool is applied to determine the risk level of the equipment, according on the study of equipment failures, their severity, occurrence probability and failure detectability. The methodology was applied to a high-pressure polyurethane injection machine; for this the work was divided into two parts. The first part was based on the Analysis and Performance Determination and its operational context within the production line, and the second part the equipment failure modes is determined, and based on them the risk of failures and failure modes was determined in a Number of Risk Priority. Giving us as result, 7 failure modes of the 16 analyzed, have a potential risk of happening, and can directly affect the performance of the equipment's operational context. Therefore maintenance activities with their respective priority are established. It was found that 7 of the 16 failure modes are potential main causes

\footnotetext{
${ }^{1}$ Escuela Superior Politécnica de Chimborazo, Riobamba, Ecuador. dlarrea@espoch.edu.ec

${ }^{2}$ Escuela Superior Politécnica de Chimborazo, Riobamba, Ecuador. david.redroban@espoch.edu.ec

${ }^{3}$ Escuela Superior Politécnica de Chimborazo, Riobamba, Ecuador. antoniog.castillo@espoch.edu.ec
} 
for equipment functional failure, therefore those must be prevented. The main problems of the equipment are: Partially clogged pipes, Inadequate temperature, based on the Priority Risk Number analysis, there is no electricity supply. It was determined that the tasks of Inspection and cleaning of pipe, Calibration of pressure and temperature controls, Checking the power supply and its parameters are tasks to avoid functional failures and decrease the equipment failure by $42.8 \%$.

Keywords: failure, failure mode, risk priority number; maintenance tasks, high pressure polyurethane injection machine.

\section{Resumen.}

La priorización de las actividades es muy importante para saber la importancia de ejecutar una actividad de mantenimiento. La metodología del Análisis de Modos y Efectos de Fallo (AMEF) es una herramienta que permite priorizar la atención del mantenimiento para los equipos; esta herramienta en la actualidad se la aplica para determinar el nivel de riesgo de los equipos en base al estudio de las fallas de los equipos, su severidad, probabilidad de ocurrencia y la detectabilidad de la falla. La metodología se aplicó a una máquina de inyección de poliuretano de alta presión; para esto el trabajo se dividió en dos partes, la primera se basó en el Análisis y Determinación del funcionamiento y su contexto operacional dentro de la línea de producción, y la segunda parte se determinaron los modos de falla de los equipos y en base a ellos se determinó en Número de Prioridad de Riesgo de las fallas y sus modos de falla, dándonos como resultado que 7 modos de falla de los 16 analizados, tienen un riesgo potencial de ocurrencia y pueden afectar directamente al desempeño del contexto operacional del equipo, por lo que se establecen actividades de mantenimiento con su respectiva prioridad. Se encontró que 7 de los 16 modos de falla son potenciales causas principales para el fallo funcional del equipo por lo que se deben prevenir. Los principales problemas del equipo son: Cañerías Parcialmente obstruidas; Temperatura inadecuada; No existe suministro de electricidad en base al análisis del Número Prioritario de Riesgo. Se determinaron que las tareas de Inspección y limpieza de cañerías; Calibración de los controles de presión y temperatura; Revisar la alimentación eléctrica y sus parámetros son tareas para evitar fallas funcionales y disminuir en un $42.8 \%$ de probabilidad que el equipo falle.

Palabras claves: falla, modos de falla, numero de prioridad de riesgo, tareas de mantenimiento, máquina de inyección de poliuretano de alta presión.

\section{Introducción.}

Las últimas dos décadas fueron testigos de importantes avances en el desarrollo de nuevas estrategias de mantenimiento. El progreso en el área de mantenimiento ha sido motivado por el aumento en el número, tamaño, complejidad y variedad de activos físicos; creciente conciencia del impacto del mantenimiento en el medio ambiente, la seguridad del personal, la rentabilidad del negocio y la calidad de los productos. (Haddara, 2003), el mantenimiento 
se ha dedicado a realizar planes de mantenimiento adecuados, empleando diferentes técnicas y herramientas.

Hay que considerar que es necesario priorizar las actividades que se llevan a cabo en un equipo en base al análisis de riesgos ya que esto permite realizar la Planificación del mantenimiento, optimizando el plan de mantenimiento para reducir la probabilidad de los fallos que sobrepasan el criterio de aceptación, reduciendo así su riesgo. (Ltd, 2013)

Para ello se pueden emplear diferentes técnicas que permiten estimar el riesgo, una de estas herramientas es el AMEF que Una herramienta excelente para identificar la importancia del Diseño o Características del Proceso y Funciones relacionadas con las fallas de efectos. (Carrasco, s.f.)

Una máquina de inyección de poliuretano de alta presión se emplea para resolver problemas como por ejemplo de aislamiento térmico y acústico, relleno de juntas, sellando de corrientes de aire y olores, entre otros. Se adhiere a la mayoría de los materiales de construcción, excepto películas de polietileno, siliconas y teflón, además estos productos son respetuosos con el medio ambiente, no son tóxicos y de fácil aplicación.

El mantenimiento aplicado a este equipo debe ser adecuado con el fin de alcanzar una alta disponibilidad, considerando que el equipo presenta un sin número de inconvenientes al momento de realizar su función es recomendable determinar qué actividades de mantenimiento tendría mayor prioridad frente a otras.

\section{Metodología.}

Para la priorización de las tareas de mantenimiento existen diferentes métodos y herramientas, sin embargo, para este equipo se emplea el Análisis de Modos y Efectos de Falla en una máquina de inyección de poliuretano de alta presión, la cual oferta el servicio de aislamiento térmico a buses, furgones y galpones.

Para el desarrollo de la técnica de mejora continua denominado "Análisis de Modos y Efectos de Falla" se tomó la metodología de Moubray; RCM II. Adicionalmente, se desarrolló el cálculo del número prioritario de riesgo (NPR) para la toma de la acción correctiva necesaria.

Para un buen desarrollo del análisis de riesgo del equipo se consideran los siguientes pasos:

- Definición de la función del equipo

- Determinación de fallas

- Determinación de los modos de falla

- Determinación de los efectos de falla

1. Definición de la función del equipo: La enunciación de una función debe consistir de un verbo, un objeto, y el nivel de desempeño deseado (Moubray, 2004) 
2. Determinación de fallas: Se define "falla" como la incapacidad de un bien de cumplir con las funciones que el usuario espera realice. Y define a la falla (Moubray, 2004) el autor nos menciona que existe la falla funcional y de esta se clasifican en dos tipos de fallo Falla total y parcial. Sin embargo, consideramos el desarrollo de lo que menciona la norma. La norma UNE EN 060300 menciona que los fallos funcionales incluyen (AENOR, 2013):

a) pérdida completa de la función;

b) incapacidad de satisfacer el requisito de funcionamiento;

c) función intermitente;

d) funcionamiento cuando se requiera.

3. Determinación de los modos de falla: forma en la que una pieza o conjunto pudiera fallar potencialmente a la hora de satisfacer el propósito de diseño/proceso, los requisitos de rendimiento y/o las expectativas del cliente. (ESPAÑA, 2004) por lo que se podría concluir que el modo de falla es la causa por la cual un equipo podría llegar a fallar funcionalmente.

Para facilitar la determinación de los modos de fallo, menciona Morales en su libro, se puede técnica de los 5 porqués que consiste en la exploración de un problema por medio de la causa-efecto repitiendo cinco veces la sencilla pregunta ¿Por qué? (Morales, 2013).

4. Determinación de los efectos de falla: Es necesario determinar el efecto de la falla debido al respectivo modo de falla. Es por lo que Moubray nos dice que el efecto de falla describe lo que pasa cuando ocurre un modo de falla. (Moubray, 2004).

5. Determinación del estado actual: En este punto se debe considerar el cálculo del Número Prioritario de riesgo (NPR) o conocido también como Índice de Prioridad de Riesgo (IPR). Es necesario considerar las ponderaciones de Severidad (Gravedad, Según otros autores), Ocurrencia (Frecuencia) y Detección (Detectabilidad).

La ocurrencia se clasifica según la probabilidad de falla, que representa el número relativo de fallas anticipadas durante la vida de diseño del artículo. (Peldez, 1995)

La gravedad se clasifica según la gravedad del efecto del modo de falla. Los efectos de un modo de falla normalmente se describen por los efectos en el usuario del producto o como los vería el usuario. Por ejemplo, algunos efectos de falla comunes para los automóviles son: ruido excesivo, operación intermitente, control deficiente y manejo brusco. (Peldez, 1995) 
La capacidad de detección es una evaluación de la capacidad de un programa de verificación de diseño propuesto para identificar una debilidad potencial antes de que la pieza o el ensamblaje salgan a producción. (Peldez, 1995)

El cuadro de clasificación de tal índice debería diseñarlo cada empresa en función del producto, servicio, proceso en concreto. Generalmente el rango es con números enteros, en la tabla adjunta la puntuación va del 1 al 10, aunque a veces se usan rangos menores (de 1 a 5) (ESPAÑA, 2004). Es necesario que las ponderaciones se ajusten a la realidad de cada empresa y a la importancia que le enfoquen los departamentos. En este caso se aplicaron las siguientes ponderaciones:

Tabla 1. Clasificación de la gravedad del modo fallo según la repercusión en el cliente/usuario

\begin{tabular}{|c|c|}
\hline \multicolumn{2}{|r|}{ Severidad (S) } \\
\hline Valor & Criterio \\
\hline 1 & $\begin{array}{l}\text { No es razonable esperar que este fallo de pequeña importancia } \\
\text { origine efecto real alguno sobre el rendimiento del sistema. } \\
\text { Probablemente, el cliente ni se daría cuenta del fallo. }\end{array}$ \\
\hline $2-3$ & $\begin{array}{l}\text { El tipo de fallo originaria un ligero inconveniente al cliente. } \\
\text { Probablemente, éste observara un pequeño deterioro del } \\
\text { rendimiento del sistema sin importancia. Es fácilmente } \\
\text { subsanable }\end{array}$ \\
\hline $4-6$ & $\begin{array}{l}\text { El fallo produce cierto disgusto e insatisfacción en el cliente. } \\
\text { El cliente observará deterioro en el rendimiento del sistema }\end{array}$ \\
\hline $7-8$ & $\begin{array}{l}\text { El fallo puede ser crítico y verse inutilizado el sistema. Produce } \\
\text { un grado de insatisfacción elevado. }\end{array}$ \\
\hline $9-10$ & $\begin{array}{l}\text { Modalidad de fallo potencial muy crítico que afecta el } \\
\text { funcionamiento de seguridad del producto o proceso y/o } \\
\text { involucra seriamente el incumplimiento de normas } \\
\text { reglamentarias. } \mathrm{Si} \text { tales incumplimientos } \\
\text { corresponde un } 10\end{array}$ \\
\hline
\end{tabular}

Fuente: Elaboración propia.

Tabla 2. Clasificación de la frecuencia/ probabilidad de ocurrencia del modo de fallo

\section{Ocurrencia $(\mathbf{O})$}

Valor Criterio 
Ningún fallo se asocia a procesos casi idénticos, ni se ha dado nunca en el pasado, pero es concebible.

Fallos aislados en procesos similares o casi idénticos. Es

2-3 razonablemente esperable en la vida del sistema, aunque es poco probable que suceda.

Defecto aparecido ocasionalmente en procesos similares o

4-5 previos al actual. Probablemente aparecerá algunas veces en la vida del componente/sistema.

6-8

El fallo se ha presentado con cierta frecuencia en el pasado en procesos similares o previos procesos que han fallado.

$9-10$

Fallo casi inevitable. Es seguro que el fallo se producirá frecuentemente.

Fuente: Elaboración propia.

Tabla 3. Clasificación de la facilidad de detección del modo de fallo

\begin{tabular}{cl}
\hline Valor & \multicolumn{1}{c}{$\begin{array}{c}\text { Detección (D) } \\
\text { Criterio }\end{array}$} \\
\hline 1 & $\begin{array}{l}\text { El defecto es obvio. Resulta muy improbable que } \\
\text { no sea detectado por los controles existentes } \\
\text { El defecto, aunque es obvio y fácilmente } \\
\text { detectable, podría en alguna ocasión escapar a un } \\
\text { primer control, aunque sería detectado con toda } \\
\text { seguridad a posteriori. }\end{array}$ \\
& $\begin{array}{l}\text { El defecto es detectab le y posiblemente no } \\
\text { llegue al cliente. Posiblemente se detecte en los } \\
\text { últimos estadios de producción }\end{array}$ \\
& $\begin{array}{l}\text { El defecto es de tal naturaleza que resulta difícil } \\
\text { detectarlo con los procedimientos establecidos } \\
\text { hasta el momento } \\
\text { El defecto no puede detectarse. Casi seguro que } \\
\text { lo percibirá el cliente final. }\end{array}$ \\
\hline
\end{tabular}

Fuente: Elaboración propia. 
Una vez que se evalúan los índices de criticidad en base a los factores de Severidad, Probabilidad de Ocurrencia y Probabilidad de detección, La estimación de la significancia

se realizó calculando el IR a través de la siguiente: (Cartín-Rojas, Villarreal, \& Morera, 2014)

fórmula:

$\mathrm{IR}=\mathrm{S} \times \mathrm{O} \times \mathrm{D}$

Donde:

$\mathrm{S}=$ Severidad del riesgo

$\mathrm{O}=$ Probabilidad de ocurrencia

$\mathrm{D}=$ Probabilidad de detección

En la actualidad surgen propuestas para mejorar los criterios de priorización del AMEF tradicional, mediante varios enfoques como:

- $\quad \operatorname{RPR}(1-1,000)$

Se ha definido una nueva escala para la priorización del riesgo, que se extiende más allá de la RPR convencional. Los números enteros del 1 al 1,000 se usan para representar el riesgo creciente de las 1,000 combinaciones posibles de detección de ocurrencia de gravedad, llamadas RPR. Las 1,000 combinaciones son tabuladas por un experto para aumentar el riesgo. Las fallas que tienen un rango más alto se indican como las más importantes y se les da una prioridad más alta que las que tienen un rango más bajo. La caracterización de este sistema se basa en el conocimiento experto, generalmente en forma de reglas "If-Then", que se implementan fácilmente mediante declaraciones condicionales. (Prabhu, 2000)

- Números de prioridad de riesgo difusos (FRPN) para la priorización de los modos de falla, definido como la media geométrica ponderada difusa de los valores difusos determinados para los tres factores ( $\mathrm{S}, \mathrm{O}$ y $\mathrm{D})$, calculados utilizando conjuntos de nivel alfa y modelos de programación lineal. El número FRPN, utiliza un método de centroides basada en conjuntos de niveles alfa. (Wang, 2007)

6. Determinación de acciones correctoras (Mantenimiento): Se describirá en este apartado la acción correctora propuesta. Generalmente el tipo de acción correctora que elegiremos seguirá los siguientes criterios, de ser posible: (ESPAÑA, 2004)

- Cambio en el diseño del producto, servicio o proceso general.

- Cambio en el proceso de fabricación.

- Incremento del control o la inspección 
A partir de las acciones correctoras consideradas se podrá emplear el mantenimiento Correctivo y Preventivo dependiendo del componente o item relacionado al modo de falla y su Prioridad.

Hay que considerar que el mantenimiento correctivo se realiza después del reconocimiento de una avería y que está destinado a poner a un elemento en un estado en que pueda realizar una función requerida. (AENOR, Terminología del Mantenimiento, 2018)

Mientras que el mantenimiento Preventivo es llevado a cabo para evaluar y/o mitigar la degradación y reducir la probabilidad de fallo de un elemento. (AENOR, Terminología del Mantenimiento, 2018), teniendo en cuenta sus definiciones se podrá establecer un plan de mantenimiento.

Este último no es más que un documento que contiene el conjunto de tareas de mantenimiento programado que debemos realizar en una planta para asegurar los niveles de disponibilidad que se hayan establecido. (Garrido, 2003)

Las tareas de mantenimiento indican qué acción, o conjunto de acciones, debe realizar un técnico de mantenimiento para completar una orden de trabajo. (Aprendizaje, 2020)

\section{Discusión y resultados.}

La priorización de las actividades de mantenimiento es necesaria para poder combatir o mitigar los riesgos y los efectos que se desencadenen debido a una falla funcional sea esta total o parcial.

Las tareas priorizadas se encontraron de la siguiente manera:

\section{Definición de la función del equipo:}

Tabla 4. Parámetros técnicos de la máquina.

\begin{tabular}{lc}
\hline \multicolumn{1}{c}{ Modelo } & $\begin{array}{c}\text { JBW-A200 Polyurethane Spray } \\
\text { Machine }\end{array}$ \\
\hline Máxima presión de trabajo & $15 \mathrm{Mpa}$ \\
Longitud máxima de cañería & $60 \mathrm{~m}$ \\
Temperatura máxima del fluido & $80^{\circ} \mathrm{C}$ \\
Caudal máximo de salida & $8 \mathrm{~kg} / \mathrm{min}$ \\
Presión de aire requerida & $0,5-0,8 \mathrm{Mpa} 1 \mathrm{~m} 3 / \mathrm{min}$ \\
Potencia & $8000 \mathrm{~W}$ \\
Voltaje & Una fase $220 \mathrm{~V} / 50 \mathrm{~Hz} / 35 \mathrm{~A}$ \\
& Tres fases $380 \mathrm{~V} / 50 \mathrm{~Hz} / 20 \mathrm{~A}$ \\
\hline
\end{tabular}


Dimensiones de la máquina

$(550 \times 700 \times 1200) \mathrm{mm}$

Peso neto $150 \mathrm{~kg}$

Fuente: Elaboración propia.

Figura 1. Máquina de inyección de poliuretano de alta presión.

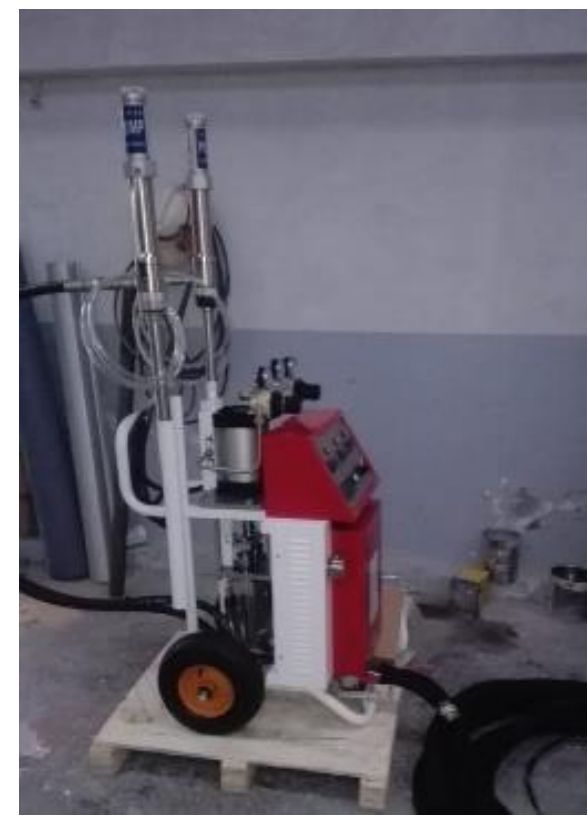

Fuente: Empresa de Sellado de carrocerías

En base a las diferentes fuentes con que se cuenta como experiencia de los trabajadores, catálogos y el equipo de mantenimiento se pudo establecer La función principal de la máquina de Inyección de Poliuretano:

- Inyectar de forma continua espuma de poliuretano suave o rígido durante la producción de aislamientos térmicos.

\section{Determinación de fallas:}

Teniendo en cuenta las diferentes fuentes de información y considerando que las fallas funcionales pueden ser totales o parciales, se encontró un listado de fallos estos fallos son los que frecuentemente suelen suceder en el equipo:

- Consideramos cuando la Inyectora es Totalmente incapaz de inyectar de forma continua espuma de poliuretano suave o rígida durante la producción de aislamientos térmicos.

- Cuando la máquina Inyecta espuma de poliuretano en niveles inferiores de los estándares de funcionamientos elegidos por el usuario. 


\section{Determinación de los modos de falla:}

Teniendo en cuenta que los modos de falla son las causa para que aparezcan las fallas, sen encontró los siguientes modos de falla según cada fallo:

Falla Total: Consideramos cuando la Inyectora es Totalmente incapaz de inyectar de forma continua espuma de poliuretano suave o rígida durante la producción de aislamientos térmicos.

\section{Modos de fallo:}

- No existe suministro de electricidad.

- Sistema electrónico descalibrado.

- No hay Presión de trabajo.

- Válvula de reguladora de presión con fugas.

- Válvula reguladora de presión tiene desgastados los asientos y no sella.

- Fugas de calor en el calentador.

- Potencia inadecuada suministrada a la varilla de calentamiento.

- Boquilla de la pistola obstruida.

Falla Parcial: la máquina Inyecta espuma de poliuretano en niveles inferiores de los estándares de funcionamientos elegidos por el usuario

\section{Modos de Fallos:}

- Presión inadecuada de trabajo.

- Válvula reguladora de presión tiene desgastados los asientos y no sella.

- Cañerías Parcialmente obstruidas.

- El suministro de energía no es el requerido.

- Suministro de energía intermitente.

- No se activan las alarmas del equipo.

- Temperatura inadecuada.

\section{Determinación de los efectos de falla:}

Los efectos de falla como sabemos indican como nos damos cuenta de que una falla se ha presentado por un modo de falla específico, es por esto que una vez que se encontró los modos de falla se procede a determinar los efectos de que existan esos modos de fallo.

Tabla 5. Modos de Falla y Sus efectos

\section{Modos de fallo}

No existe suministro de electricidad
Efectos de fallo

El equipo no enciende 
Sistema electrónico descalibrado

No hay Presión de trabajo

Válvula de reguladora de presión con fugas.

Válvula reguladora de presión tiene desgastados los asientos y no sella

Fugas de calor en el calentador

Potencia inadecuada suministrada a la varilla de calentamiento

Boquilla de la pistola obstruida

Presión inadecuada de trabajo

Válvula reguladora de presión tiene desgastados los asientos y no sella

Cañerías Parcialmente obstruidas

El suministro de energía no es el requerido

Suministro de energía intermitente

No se activan las alarmas del equipo
El equipo enciende normalmente pero no se puede trabajar debido a que no se inyecta el suministro de aire adecuado ni la temperatura es la requerida El equipo no funciona debido a a que no se presuriza el líquido utilizado

Se puede notar la presencia de fugas por el cuerpo o sellos de la válvula, perdiendo presión que es notable en los indicadores de presión.

La válvula no regula la presión por lo cual no se puede presurizar el líquido a la hora de realizar el trabajo.

Se evidencia que el calor es palpable y también se nota que la pistola no alcanza la temperatura adecuada El indicador de temperatura muestra que la varilla no produce suficiente calor para ser suministrada a la pistola El suministro de pintura se va reduciendo a medida que se realiza el trabajo.

Se observa que el producto final no tiene las características adecuadas debido a la falta de presión

Se observa que el producto final no tiene las características adecuadas debido a la falta de presión

El caudal de suministro de la pintura se ve reducido considerablemente El equipo funciona de forma intermitente, observado que algunos elementos se queman o se desconectan.

El equipo funciona de forma intermitente, observado que algunos elementos se queman o se desconectan.

las diferentes alarmas del sistema de control permanecen apagadas, resultando en una condición peligrosa debido a que no se sabe si el equipo está operando o no 
ISSN: 2602-8085

La pintura suministrada no se adhiere de

Temperatura inadecuada forma adecuada a la superficie a cubrir debido a su temperatura inferior a la necesaria

Fuente: Elaboración propia.

De este modo se identificaron los efectos de fallo para considerar el riesgo y posteriormente las tareas de mantenimiento.

\section{Determinación del estado actual}

La evaluación de la situación actual del equipo es donde se evidencia si la empresa ha considerado tareas de mantenimiento y las ha aplicado al equipo con anterioridad.

Adicional a esto se procede a determinar el NPR, por sus siglas en inglés (Risk Priority Number), es el producto de multiplicar la severidad, la ocurrencia, y la detección o detectabilidad. El NPR es un número entre 1 y 1000 que nos indica la prioridad que se le debe dar a cada falla para eliminarla. Cuando el RPN es superior a 100 es un claro indicador de que deben implementarse acciones de prevención o corrección para evitar la ocurrencia de las fallas, de forma prioritaria. Sin embargo, el objetivo general es el de tratar todas las fallas; muchos expertos coinciden en que un RPN superior a 30 requiere de un despliegue enfocado en el tratamiento del modo de falla. (MOLINA, 2018)

Tabla 6. Estado Actual del equipo y Evaluación de Riesgos.

\begin{tabular}{|c|c|c|c|c|c|c|c|}
\hline $\begin{array}{c}\text { Time is it hello } \\
\text { hello }\end{array}$ & Modos de fallo & Efectos de fallo & $\begin{array}{c}\text { Medidas de } \\
\text { ensayo y } \\
\text { control } \\
\text { previstas }\end{array}$ & S & $\mathrm{O}$ & $\mathrm{D}$ & NPR \\
\hline A. 1 & $\begin{array}{l}\text { No existe } \\
\text { suministro de } \\
\text { electricidad }\end{array}$ & $\begin{array}{l}\text { El equipo no } \\
\text { enciende }\end{array}$ & Ninguna & 8 & 4 & 4 & 128 \\
\hline A. 2 & $\begin{array}{l}\text { Sistema } \\
\text { electrónico } \\
\text { descalibrado }\end{array}$ & $\begin{array}{l}\text { El equipo enciende } \\
\text { normalmente pero } \\
\text { no se puede trabajar } \\
\text { debido a que no se } \\
\text { inyecta el suministro } \\
\text { de aire adecuado ni } \\
\text { la temperatura es la } \\
\text { requerida }\end{array}$ & Ninguna & 8 & 2 & 4 & 64 \\
\hline
\end{tabular}


El equipo no

A.3

A. 4

A.5

A. 6

A.7

A. 8

B.1
No hay Presión funciona debido a de trabajo que no se presuriza el líquido utilizado

Se puede notar la presencia de fugas

Válvula de por el cuerpo o reguladora de sellos de la válvula, presión con perdiendo presión fugas. que es notable en los indicadores de presión.

Válvula La válvula no regula reguladora de la presión por lo cual presión tiene no se puede desgastados los presurizar el líquido asientos y no a la hora de realizar sella

el trabajo.

Se evidencia que el Fugas de calor calor es palpable y en el también se nota que calentador.

el la pistola no alcanza la temperatura adecuada

El indicador de Potencia temperatura muestra inadecuada que la varilla no suministrada a produce suficiente la varilla de calor para ser calentamiento suministrada a la pistola

El suministro de Boquilla de la pintura se va pistola reduciendo a medida obstruida que se realiza el trabajo.

Presión

Se observa que el inadecuada de producto final no
Ninguna $\quad \begin{array}{llll}8 & 2 & 4 & 64\end{array}$ trabajo
Ninguna $\quad \begin{array}{llll}6 & 2 & 4 & 48\end{array}$

$\begin{array}{lllll}\text { Ninguna } & 6 & 2 & 1 & 12\end{array}$

$\begin{array}{lllll}\text { Ninguna } & 8 & 1 & 8 & 64\end{array}$

$\begin{array}{lllll}\text { Ninguna } & 8 & 1 & 8 & 64\end{array}$

$\begin{array}{lllll}\text { Ninguna } & 4 & 7 & 4 & 112\end{array}$
Ninguna $\quad \begin{array}{llll}6 & 2 & 4 & 48\end{array}$ características 
adecuadas debido a

la falta de presión

Válvula Se observa que el reguladora de producto final no presión tiene tiene las desgastados los características asientos y no adecuadas debido a sella

Cañerías la falta de presión El caudal de

B.3

B.4

B.5

B.6

B.7 obstruidas reducido

considerablemente

El equipo funciona de forma

El suministro intermitente, de energía no es observado que el requerido algunos elementos

se queman o se desconectan.

El equipo funciona de forma Suministro de intermitente, energía observado que intermitente algunos elementos se queman o se desconectan.

las diferentes alarmas del sistema de control No se activan las alarmas del equipo.

permanecen

apagadas, resultando en una condición peligrosa debido a que no se sabe si el equipo está operando o no

Temperatura inadecuada
La pintura suministrada no se $\quad$ Ninguna $\quad \begin{array}{lllll}7 & 5 & 5 & 175\end{array}$ $\begin{array}{lllll}\text { Ninguna } & 6 & 2 & 1 & 12\end{array}$

$\begin{array}{lllll}\text { Ninguna } & 5 & 7 & 5 & 175\end{array}$

Ninguna $\quad \begin{array}{llll}5 & 6 & 4 & 120\end{array}$

$\begin{array}{lllll}\text { Ninguna } & 5 & 6 & 4 & 120\end{array}$ adhiere de forma 
adecuada a la

superficie a cubrir

debido a su

temperatura inferior

a la necesaria

Fuente: Elaboración propia.

\section{Determinación de acciones correctoras (Mantenimiento):}

Una vez que se ha determinado el Índice de Riesgo de cada modo de fallo, se deben ordenar los valores para poder estructurar las tareas de las que tienen mayor riesgo, hasta las de menor riesgo, de este modo se establecerá que tipo de mantenimiento se aplicará, teniendo como resultado:

De la determinación de $\mathbf{1 6}$ modos de falla en el equipo existen, 7 modos de falla con un índice de prioridad de riesgo alto.

Mientras que, durante el cálculo del NPR se obtuvieron valores elevados y pequeños, pero se debe tomar en cuenta que, los números elevados son a los cuales se pone más énfasis a la hora de realizar acciones correctivas.

Para definir las acciones de mayor a menor importancia se definió una escala, como se describe a continuación:

- NPR>100: se toman acciones correctivas que deben ser realizadas con la mayor prontitud.

- 50>NPR<100: se toman acciones correctivas que van a ser realizadas con una mediana prontitud.

- $\quad \mathrm{NPR}$ <50: se toman acciones correctivas que deben ser realizadas, pero en orden de criticidad son bajas.

Con estos datos se determinan las actividades de mantenimiento que prevendrán o corregirán los modos de fallo determinados, como resultado tenemos:

Tabla 7. Acciones correctoras en base al Riesgo.

\begin{tabular}{cccc}
\hline & Modos de fallo & Npr & Acción correctora \\
\hline B.3 & Cañerías Parcialmente obstruidas & 175 & $\begin{array}{c}\text { Inspección y limpieza de } \\
\text { cañerías }\end{array}$ \\
B.7 & Temperatura inadecuada & 150 & $\begin{array}{c}\text { Calibrar adecuadamente los } \\
\text { controles de presión y } \\
\text { temperatura }\end{array}$ \\
\hline
\end{tabular}


A. 1

No existe suministro de electricidad

B.4

El suministro de energía no es el requerido

B.5 Suministro de energía intermitente

A.8 Boquilla de la pistola obstruida

A.2 Sistema electrónico descalibrado

A.3 No hay Presión de trabajo

A.6 Fugas de calor en el calentador.

A. 7

Potencia inadecuada suministrada a la varilla de calentamiento

Válvula de reguladora de presión

A. 4

con fugas.

B.1 Presión inadecuada de trabajo

Válvula reguladora de presión

A.5 tiene desgastados los asientos y no sella

Válvula reguladora de presión

B.2 tiene desgastados los asientos y no sella

No se activan las alarmas del equipo.
128

120

120

112 Revisar el estado de las boquillas

Regular los dispositivos que

64

64

Inspeccionar la entrada de aire y su almacenamiento

Revisar el estado de los aisladores térmicos en búsqueda de condiciones anormales de funcionamiento

Revisar el estado de los sensores y controladores de temperatura

Revisión del estado de la válvula reguladora en búsqueda de condiciones anormales.

Verificar y calibrar la presión de trabajo y de ser necesario cambiar sellos

Pruebas de funcionamiento de la 12 válvula para accionar el flujo del fluido

Verificar el funcionamiento de la 12 válvula de presión/ Calibración de la válvula de presión Ajuste de elementos eléctrico / 8 Reemplazo de elementos de ser necesario

Fuente: Elaboración propia.

Se Puede observar que: La Inspección y limpieza de cañerías; Calibrar adecuadamente los controles de presión y temperatura; Revisar la alimentación eléctrica y sus parámetros, son 
tareas que disminuirían un $42.82 \%$ de probabilidad de que el equipo presente fallas funcionales.

\section{Conclusiones.}

- Se encontró que 7 de los 16 modos de falla son potenciales causas principales para el fallo funcional del equipo por lo que se deben prevenir.

- Los modos de falla que representan mayor riesgo para el funcionamiento del equipo son: Cañerías Parcialmente obstruidas; Temperatura inadecuada; No existe suministro de electricidad en base al análisis del Número Prioritario de Riesgo.

- La Inspección y limpieza de cañerías; Calibrar adecuadamente los controles de presión y temperatura; Revisar la alimentación eléctrica y sus parámetros son las tareas más importantes que se deben llevar a cabo en el equipo para evitar fallas funcionales y así disminuir en un $42.8 \%$ de probabilidad de que el equipo falle.

- La priorización de las actividades de mantenimiento es necesarias en los sistemas que brindan un servicio por tiempos continuos de modo que estén disponibles todo el tiempo.

- El análisis de Modos y Efectos de fallo es una herramienta amigable de emplear siempre y cuando se cuente con los datos técnicos necesarios para emplearlo. Y el criterio del personal fundamentado en la herramienta.

\section{Referencias bibliográficas.}

AENOR. (2013). Gestión de la confiabilidad. Parte 3-11: Guía de aplicación; Mantenimiento Centrado en la Fiabilidad. España, España.

AENOR. (Julio de 2018). Terminología del Mantenimiento. España.

Aprendizaje, C. d. (2020). Tipos de tareas de mantenimiento. Obtenido de UpKeep: https://www.onupkeep.com/learning/maintenance-terms/maintenancetasks\#: :text=Maintenance\%20tasks\%20indicate\%20which\%20action,of\%20equip ment $\% 20$ that $\% 20$ needs $\% 20$ maintained.

Carrasco, U. (s.f.). Modos de Falla \& Análisis de Efectos. Obtenido de Academia: https://www.academia.edu/37821847/FMEA_AMEF_Modos_de_Falla_and_An\%C 3\%A1lisis_de_Efectos

Cartín-Rojas, A., Villarreal, A., \& Morera, A. (2014). Implementación del análisis de riesgo en la industria alimentaria mediante la metodología AMEF: enfoque práctico y conceptual. SCielo, 16.

ESPAÑA, M. D. (2004). NTP 679: Análisis modal de fallos y efectos. AMFE. Obtenido de https://www.insst.es/documents/94886/326775/ntp_679.pdf/3f2a81e3-531c-4daabfc2-2abd3aaba4ba 
Garrido, S. G. (2003). Organización y gestión integral de mantenimiento. Madrid: Díaz de Santos, S. A.

Haddara, F. I. (2003). Risk-based maintenance (RBM): a quantitative approach for maintenance/inspection scheduling and planning. Journal of Loss Prevention in the Process Industries, 13.

Ltd, A. (13 de Julio de 2013). AlterEvo Ltd. Obtenido de http://alterevoingenieros.blogspot.com/2013/07/mantenimiento-basado-en-riesgo-lamas.html\#: :text=La\%20norma\%20Norsok\%20Standard\%20Z,repuestos\%20y\%20 su\%20localizaci\%C3\%B3n.

MOLINA, G. I. (2018). ANÁLISIS DE MODOS Y EFECTOS DE FALLAS EN LOS EQUIPOS DELAVANDERÍA DEL HOSPITAL REGIONAL DE CONCEPCIÓN. Chile: UNIVERSIDAD TECNICA FEDERICO SANTA MARIA.

Moubray, J. (2004). MANTENIMIENTO CENTRADO EN LA CONFIABILIDAD. United Kingdom: Aladon Ltd.

Peldez, J. B. (1995). Fuzzy logic prioritization of failures in a system failure mode, effects and criticality. ELSEVIER, 11.

Prabhu, N. R. (2000). Modified approach for prioritization of failures in a system failure mode and effects analysis. International Journal of Quality \& Reliability Management, 12.

Wang, Y.-M. (2007). Risk evaluation in failure mode and effects analysis using fuzzy weighted geometric mean. ScienceDirect, 13.

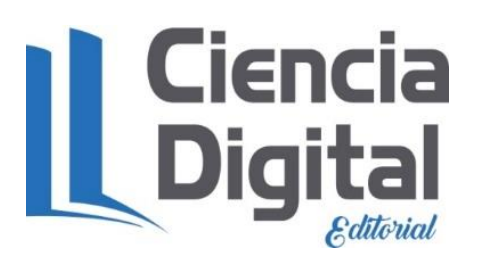




\section{PARA CITAR EL ARTÍCULO INDEXADO.}

Larrea Moreano, A. D., Redrobán Dilon, C. D., \& Castillo Medina, A. G. (2020). Priorización del mantenimiento mediante la determinación del número prioritario de riesgo, y el análisis de modos y efectos de fallos de una máquina de inyección de poliuretano de alta presión. Ciencia Digital, 4(3), 317-335. https://doi.org/10.33262/cienciadigital.v4i3.1353

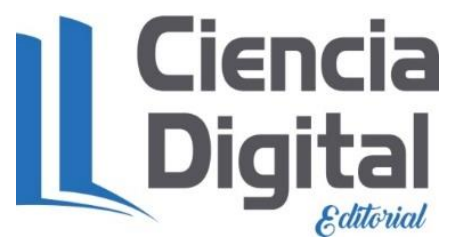

El artículo que se publica es de exclusiva responsabilidad de los autores y no necesariamente reflejan el pensamiento de la Revista Ciencia Digital.

El artículo queda en propiedad de la revista y, por tanto, su publicación parcial y/o total en otro medio tiene que ser autorizado por el director de la Revista Ciencia Digital.
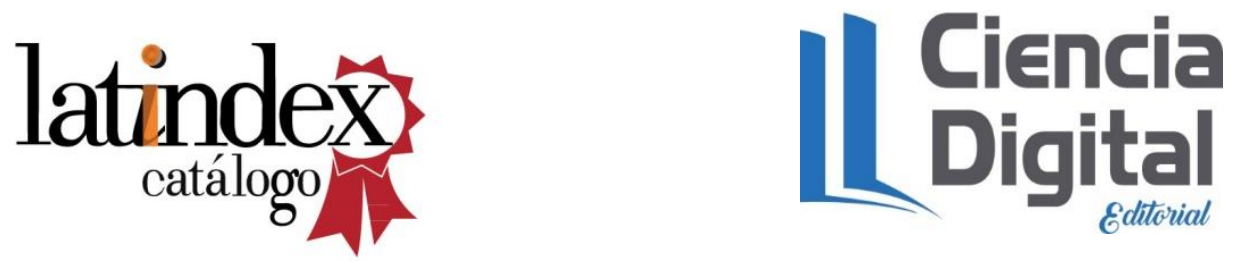